\title{
Conquiliometria de Littoraria flava (King, 1832) (Gastropoda: Littorinidae) do litoral sul brasileiro
}

\author{
Mara Rúbia Ferreira Barros ${ }^{1}$ (), Weverton John Pinheiro dos $\operatorname{Santos}^{1}$ (이 \& Rafael \\ Anaisce das Chagas ${ }^{1,2}$ (1)
}

(1) Universidade Federal Rural da Amazônia, Instituto Socioambiental e dos Recursos Hídricos, Programa de Pós-Graduação em Aquicultura e Recursos Aquáticos Tropicais, Avenida Perimetral 2501, Terra Firme 66077-830, Belém, Pará, Brasil. E-mail: eng.p.marabarros@gmail.com, weverton_john@hotmail.com

(2) Universidade Federal do Pará, Instituto de Ciências Biológicas, Programa de Pós-Graduação em Ecologia, Rua Augusto Corrêa 01, Guamá 66075-110, Belém, Pará, Brasil. E-mail: rafaelanaisce@ @otmail.com

Barros M.R.F., Santos W.J.P. \& Chagas R.A. (2020) Conquiliometria de Littoraria flava (King, 1832)

(Gastropoda: Littorinidae) do litoral sul brasileiro. Pesquisa e Ensino em Ciências Exatas e da Natureza, 4: e1328. http://dx.doi.org/10.29215/pecen.v4i0.1328

Editor acadêmico: Silvio F. B. Lima. Recebido: 31 Janeiro 2020. Aceito: 25 Março 2020. Publicado: 02 Abril 2020.

Resumo: Littoraria flava (King, 1832) é um gastrópode marinho amplamente distribuído no litoral brasileiro, habitando a zona entremarés, geralmente, aderido a rochas. Este trabalho teve como objetivo analisar a conquiliometria de L. flava com base em 129 gastrópodes coletados em um costão rochoso no litoral de Balneário Camboriú, estado de Santa Catarina, no mês de novembro de 2017. Para tanto, realizou-se relações morfométricas entre as medidas externas da concha (comprimento, largura e altura) e as medidas de abertura da concha (comprimento e largura), estimando-se também o Indicador de Estabilização da Forma (IEF) da concha. Os resultados indicaram que todas as relações morfométricas apresentaram uma tendência linear, corroboradas pelas elevadas correlações, sendo classificadas como "extremamente fortes" e alometria positiva entre a altura da concha e o comprimento da abertura, com as demais relações classificadas por negativa. Os resultados de IEF evidenciaram pequenas flutuações nas razões morfométricas de $L$. flava e, deste modo, indicaram que a espécie apresenta conquiliometria proporcional ao longo de seu crescimento. O presente trabalho apresenta dados importantes acerca da conquiliometria de $L$. flava, o que possibilita a utilização da espécie como provável organismo indicador de anomalias ambientais, a partir da análise de sua concha.

Palavras chave: Moluscos, relações morfométricas, IEF, litorinídeos.

\section{Conchometry of Littoraria flava (King, 1832) (Gastropoda: Littorinidae) from the southern coast of Brazil}

Abstract: Littoraria flava (King, 1832) is a marine gastropod widely distributed on the Brazilian coast, inhabiting the intermareal region, generally attached to rocks. The present study aims to analyze the conchiliometry of L. flava from 129 gastropods collected on a rocky shore on the coast of Balneário Camboriú, state of Santa Catarina, in the month of November 2017. For this purpose, morphometric relationships were performed between the external measures (length, width and height) of the shell and the measures of aperture (length and width) of the shell, also estimating the shape stabilization indicator (IEF) of the shell. The results indicated that all the morphometric relationships performed show a linear trend, corroborated by the high correlations, being classified as "extremely strong" and positive allometry between the height of shell and the length of aperture, with the other relationships classified as negative. The results of the IEF indicated small fluctuations in the morphometric ratios of $L$. flava and, therefore, indicated that the species presents a development in the shape of the proportional conchometry throughout its growth. This work presents important data about the conchiliometry of L. flava, which makes it possible to use the species as probable indicator organism of environmental anomalies from the analysis of its shell.

Key words: Mollusks, morphometric relationships, IEF, litorinids. 
Os gastrópodes da família Littorinidae Children, 1834, popularmente conhecidos por litorinídeos, habitam a zona costeira em áreas de mangue e costões rochosos (Rios 2009). O grupo apresenta inúmeras características fenotípicas (e.g., polimorfismo de cores e morfologia da concha) devido a distribuição em vários ambientes (Simone 1998; Moutinho \& Alves-Costa 2000). Os litorinídeos são facilmente coletados por habitantes litorâneos, principalmente para fins de subsistência (Poutiers 2016) e para a confecção de zooartezanatos (Barros \& Chagas 2019). Uma outra peculiaridade dos litorinídeos é resistência elevada a longos períodos expostos ao ar (Simone 1998; Poutiers 2016). Por conta destas características, tais gastrópodes são comumente utilizados como bioindicadores no monitoramento de contaminação ambiental (Bauer et al. 1995; FerreiraJr et al. 2014).

No litoral brasileiro ocorrem os gêneros Echinolittorina Habe, 1956 e Littoraria Gray, 1833 (Andrade et al. 2003). Littoraria flava (King, 1832) é o litorinídeo mais comum na zona costeiras usualmente ocorrendo em áreas de supramarés sobre rochas de mar aberto ou em baías, sendo incomum em ambientes estuarinos (Moutinho \& Alves-Costa 2000; Rios 2009). A espécie possui hábito alimentar herbívoro (Meirelles \& Matthews-Cascon 2003) distribuindo-se no Oceano Atlântico oeste desde o litoral da Flórida (USA) até o Brasil. No Brasil, L. flava ocorre entre as regiões costeiras do estado do Pará e Santa Catarina (Cardoso et al. 2007; Rios 2009).

A sistemática e evolução dos litorinídeos tem sido amplamente estudadas (Janson 1982; Andrade et al. 2003; Reid et al. 2006; Reid et al. 2012). Além disso, o grupo tem sido utilizado como modelo em estudos morfométricos (Chapman 1995; Walker \& Grahame 2011; Doyle et al. 2018). No entanto, estudos relacionados ao gastrópode Littoraria flava ainda são escassos, sendo abordados, mais recentemente, aspectos da morfológica interna (Simone 1998), estrutura radular (Meirelles \& Matthews-Cascon 2003), padrões de agregação (Moutinho \& Alves-Costa 2000), comportamento e locomoção (Vianna et al. 2014) e aspectos reprodutivos (Cardoso et al. 2007; Ferreira-Jr et al. 2014).

Partindo do exposto, o presente estudo tem como objetivo analisar a conquiliometria de Littoraria flava com base em indivíduos coletados em costão rochoso no litoral de Balneário Camboriú, estado de Santa Catarina, região Sul do Brasil.

A área de estudo limitou-se ao costão rochoso presente entre as praias "Prainha" e "Praia do Buraco”, situada em Balneário Camboriú, litoral do estado de Santa Catarina, região Sul do Brasil (Figura 1).

A amostragem de indivíduos de Littoraria flava foi realizada em novembro de 2017 com base na coleta manual após visualização direta dos gastrópodes.

Um total de 129 indivíduos de Littoraria flava foram coletados manualmente durante a maré vazante. Posteriormente, todos os gastrópodes foram fixados em etanol $70 \%$ e transportados ao Laboratório de Ecologia Bentônica da Universidade Federal Rural da Amazônia (UFRA). Parte dos indivíduos estudados estão depositados na Coleção Malacológica do Museu de Zoologia da UFRA sob o voucher MZUFRA Moll 082.

Para a análise conquiliométrica de Littoraria flava, primeiramente, mensurou-se as medidas externas (comprimento total, largura e altura da concha) e as medidas de comprimento e largura da abertura da concha (Figura 2), aferidas com o uso de um paquímetro digital (TESA Data-Direct, precisão de $0.01 \mathrm{~mm}$ ). Posteriormente, empregou-se a metodologia utilizada por Gaspar et al. (2001) para descrever aspectos morfométricos da espécie. Deste modo, utilizou-se equações lineares $(Y=a+b . X)$ para relacionar as medidas externas entre si e entre as medidas da abertura da concha.

Paralelamente, a significância das relações, através do coeficiente de correlação de Pearson $(r$ ), foi testada por um teste $t$-Student, conforme Rodrigues (2010), sendo os coeficientes linear $(a)$ e angular $(b)$ estimados pelo método dos mínimos quadrados. Além disso, classificou-se as alometrias das equações em negativa quando $b<1$, positiva quando $b>1$ e isométrica quando $b=1$. Todas as análises foram realizadas a um nível de significância de $95 \%(\alpha=0,05)$ (Zar 2010), utilizando o software PAleontological STatistics - PAST, versão 4.0 (Hammer 2020).

Para determinar o tamanho no qual a espécie atinge a forma definida da concha aplicouse o método proposto por Gil et al. (2007), que denomina-se Indicador de Estabilização da Forma (IEF) da concha. Este método constitui-se por uma análise simples e consiste em calcular as razões, expressas em porcentagem, entre as três medidas lineares da concha. Este método, proposto 


\section{Conquiliometria de Littoraria flava}

inicialmente para moluscos bivalves, também apresenta-se eficaz na comparação morfológica de gastrópode (Chagas et al. 2018a). Para tanto, separou-se os exemplares amostrados em classes de comprimento, calculadas através da regra da raiz quadrada $(k=\sqrt{n}$, onde $k$ é o número de classes e $n$ é o número gastrópodes) e o intervalo de classes calculado pela equação $I C=(L s-L i) / k$, onde $I C$ é o intervalo de classe e $L s$ e $L i$ são os maiores e menores valores de comprimento total amostrados. Calculou-se a média das medidas lineares para cada intervalo, determinando-se as seguintes razões morfométricas: $A / C, L / C$ e $A / L$, sendo $A$ a altura, $C$ o comprimento total e $L$ a largura da concha. Adicionalmente, estimou-se a razão proporcional entre a morfometria externa da concha do gastrópode e sua abertura da concha, determinando as seguintes razões morfométricas: $C a / C, C a / L, C a / A, L a / C, L a / L, L a / A$, sendo $L a$ a largura da abertura da concha e Ca o comprimento da abertura da concha.
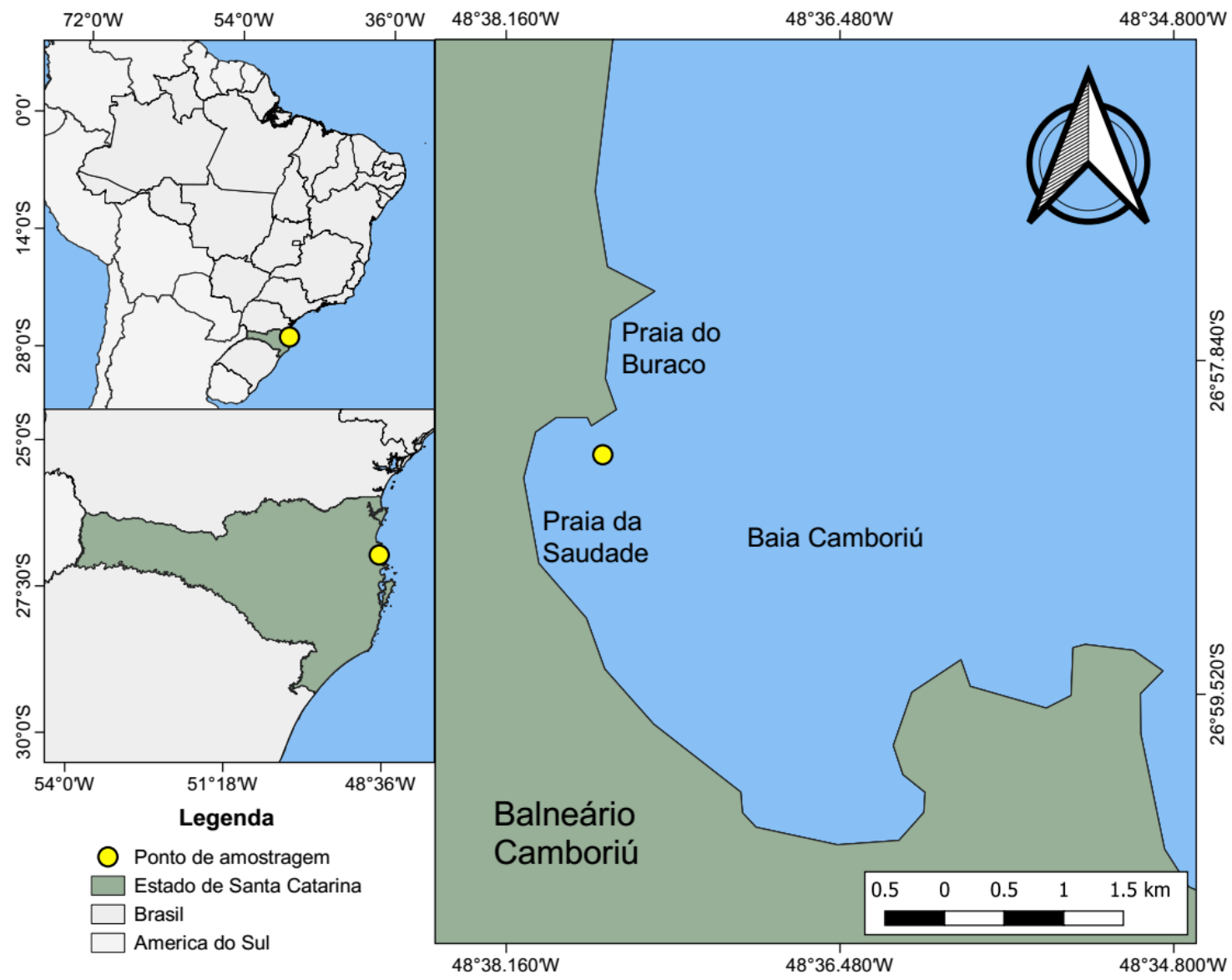

Figura 1. Localização do ponto de amostragem de indivíduos de Littoraria flava em Balneário Camboriú.

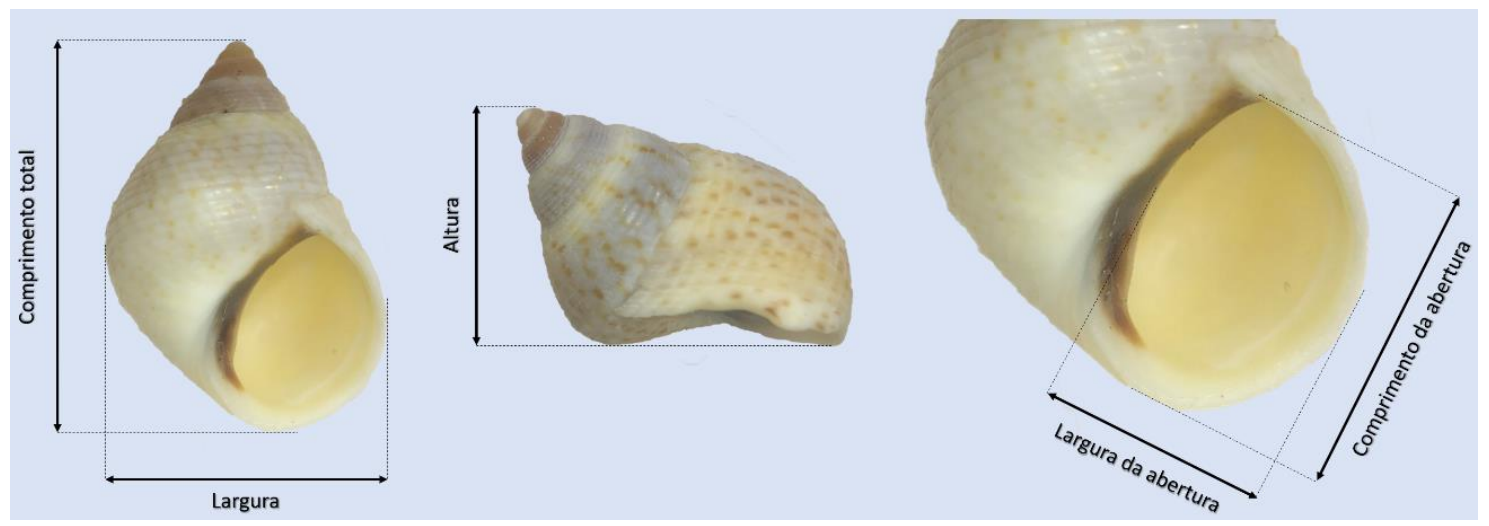

Figura 2. Medidas lineares da concha de Littoraria flava empregadas na análise conquiliométrica. 
Todas as medidas morfométricas da concha de Littoraria flava estão disponíveis na plataforma digital Data Publisher for Earth \& Environmental Science - PANGAEA (www.pangaea.de/) (Chagas et al. 2018b). Littoraria flava apresentou comprimento total de 16.12 $\pm 3.82 \mathrm{~mm}$ (Média $\pm \mathrm{DP}$ ), variando entre 3.60 e $20.51 \mathrm{~mm}$, largura de $10.46 \pm 2.47 \mathrm{~mm}$, entre 2.34 e $13.83 \mathrm{~mm}$, altura de $8.48 \pm 1.95 \mathrm{~mm}$, entre 1.97 e $10.60 \mathrm{~mm}$, comprimento da abertura de $9.52 \pm$ $2.14 \mathrm{~mm}$, entre 2.07 e $11.96 \mathrm{~mm}$ e largura da abertura de $7.31 \pm 1.65 \mathrm{~mm}$, entre 1.65 e $9.39 \mathrm{~mm}$. Ressalta-se que o comprimento total máximo registrado nesse estudo $(20.51 \mathrm{~mm})$ é superior ao registrado para a espécie, que até então era estimado no máximo de $20 \mathrm{~mm}$ (Simone 1998) (Tabela 1).

As relações morfométricas das medidas externas e da abertura da concha de Littoraria flava apresentaram uma tendência linear (Figura 3). Esse fato é corroborado pela elevada correlação das medidas morfométricas, que apresentaram coeficiente de determinação $\left(\mathrm{R}^{2}\right)$ das relações variando entre 0.879 e 0.962 . Os coeficientes de correlação de Pearson de todas as relações foram classificados como "extremamente forte" $(r>0.9)$, evidenciando a melhor relação entre o comprimento total e a altura da concha $(r=0.98)$ do gastrópode. Adicionalmente, classifica-se por alometria positiva a relação entre a altura da concha e o comprimento da abertura $(b=1.054)$, com as demais relações classificadas como negativas.

Tabela 1. Comprimento total máximo de Littoraria flava registrada no litoral brasileiro. Legenda/abreviatura: *macho e ** fêmea de L. flava; MZUSP = Museu de Zoologia da Universidade de São Paulo.

\begin{tabular}{lccl}
\hline \multicolumn{1}{c}{ Localidade (Brasil) } & CT máximo (mm) & CT médio ( \pm DP) & \multicolumn{1}{c}{ Autor } \\
\hline Balneário Camboriú - SC & 20.51 & $16.12 \pm 3.82$ & Presente estudo \\
Desconhecida (MZUSP) & 20.00 & - & Simone (1998) \\
Praia de Araçá (São Sebastião - SP) & 18.80 & $12.40 \pm 2.10$ & Moutinho \& Alves-Costa (2000) \\
Praia das Flexeiras (Itacuruçá - RJ) & $16.00^{*}$ & - & Cardoso et al. (2007) \\
Praia das Flexeiras (Itacuruçá - RJ) & $17.00^{* *}$ & - & Cardoso et al. (2007) \\
Praia de Encantadas (Ilha do Mel - PR) & 19.00 & $11.32 \pm 3.92$ & Ferreira-Jr et al. (2014) \\
\hline
\end{tabular}
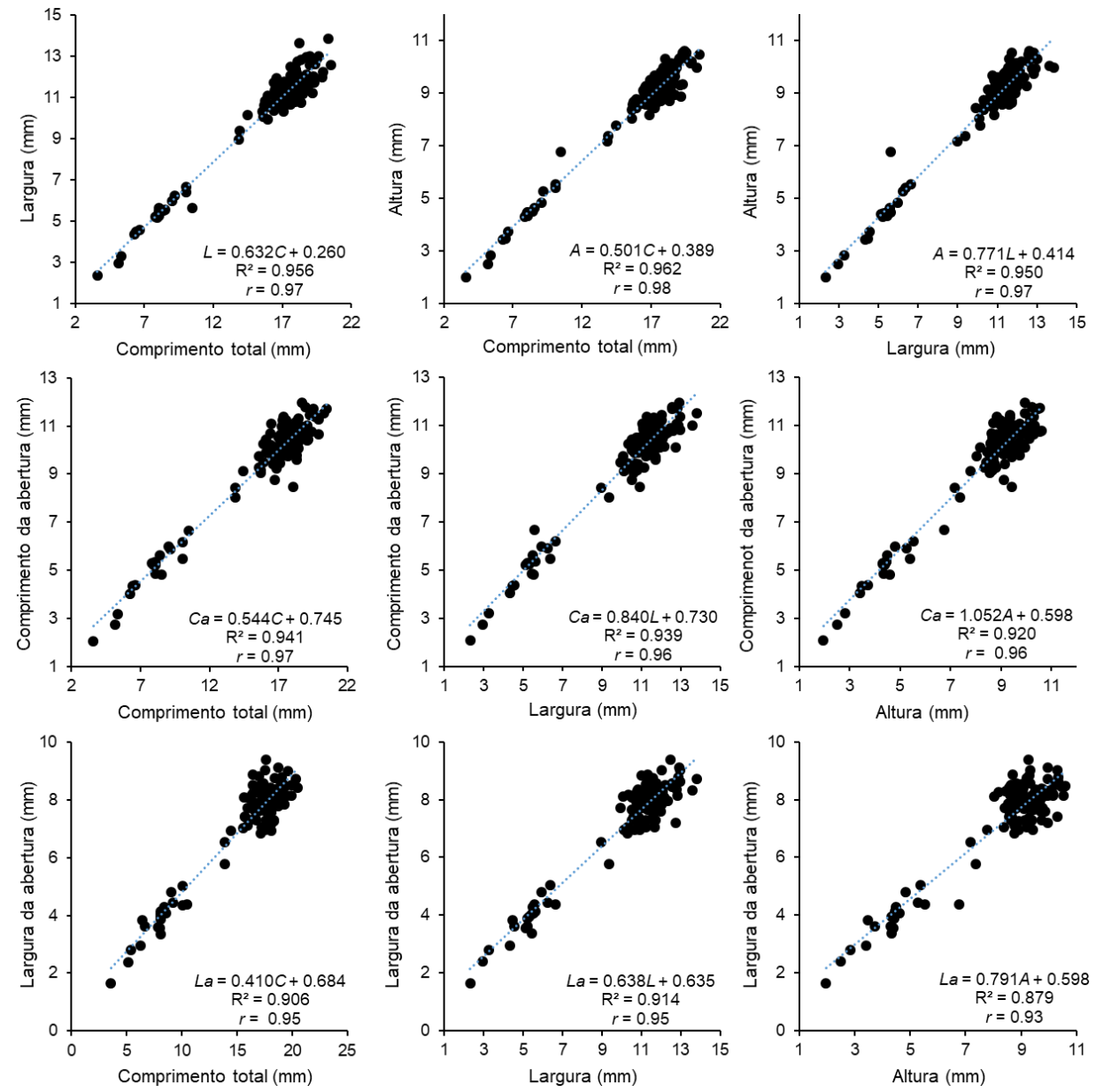

Figura 3. Relações morfométricas em conchas de Littoraria flava analisadas. 
A elevada correlação entre as variáveis encontradas em Littoraria flava neste estudo também foi evidenciada por Fletcher (1995) nas relações entre a altura e o comprimento da concha de Littorina fabalis (W. Turton, 1825) (nome válido para Littorina mariae Sacchi \& Rastelli, 1966) ( $b=0.882 ; r=0.927$ ) e indivíduos jovens e adultos de Littorina obtusata (Linnaeus, 1758) $(b=0.838$; $r=0.972$ e $b=0.838 ; r=0.972$, respectivamente). O mesmo resultado foi encontrado por Catesby \& McKillunp (1998) com Littoraria articulata (Philippi, 1846), indicando uma correlação significativa entre a largura e o comprimento da concha $(r=0.98 ; p<0.001)$.

$O$ resultado da análise de IEF indicou que Littoraria flava apresentou a menor amplitude de variação entre $L / C$, sendo a maior entre $A / L$ (Tabela 2, Figura 4). Das razões entre as medidas externas e o comprimento da abertura da concha, a menor amplitude ocorreu em $C a / A$ e a maior em $C a / L$. A amplitude das razões entre as medidas externas e a largura da abertura da concha, indicou a menor variação entre $L a / C$ e a maior entre $L a / L$. Tais resultados evidenciam pequenas flutuações nas razões morfométricas de L. flava e, deste modo, indicando que a espécie apresenta um desenvolvimento na forma da concha proporcional ao longo de seu crescimento.

Tabela 2. Razões entre as medidas externa ( $A$ a altura, $C$ o comprimento total e $L$ a largura) e da abertura da concha ( $L a$ : largura da abertura e Ca: comprimento da abertura).

\begin{tabular}{cccc}
\hline Medida morfométrica & Mínimo $(\mathrm{mm})$ & Máximo $(\mathrm{mm})$ & Média $\pm \mathrm{DP}(\mathrm{mm})$ \\
\hline$A / C$ & 0.57 & 0.69 & $0.65 \pm 0.03$ \\
$L / C$ & 0.48 & 0.58 & $0.53 \pm 0.02$ \\
$A / L$ & 1.06 & 1.29 & $1.22 \pm 0.05$ \\
$C a / C$ & 0.53 & 0.66 & $0.60 \pm 0.04$ \\
$C a / A$ & 0.88 & 0.98 & $0.93 \pm 0.04$ \\
$C a / L$ & 1.04 & 1.20 & $1.13 \pm 0.05$ \\
$L a / C$ & 0.42 & 0.54 & $0.47 \pm 0.03$ \\
$L a / A$ & 0.65 & 0.85 & $0.72 \pm 0.05$ \\
$L a / L$ & 0.78 & 0.99 & $0.88 \pm 0.06$ \\
\hline
\end{tabular}

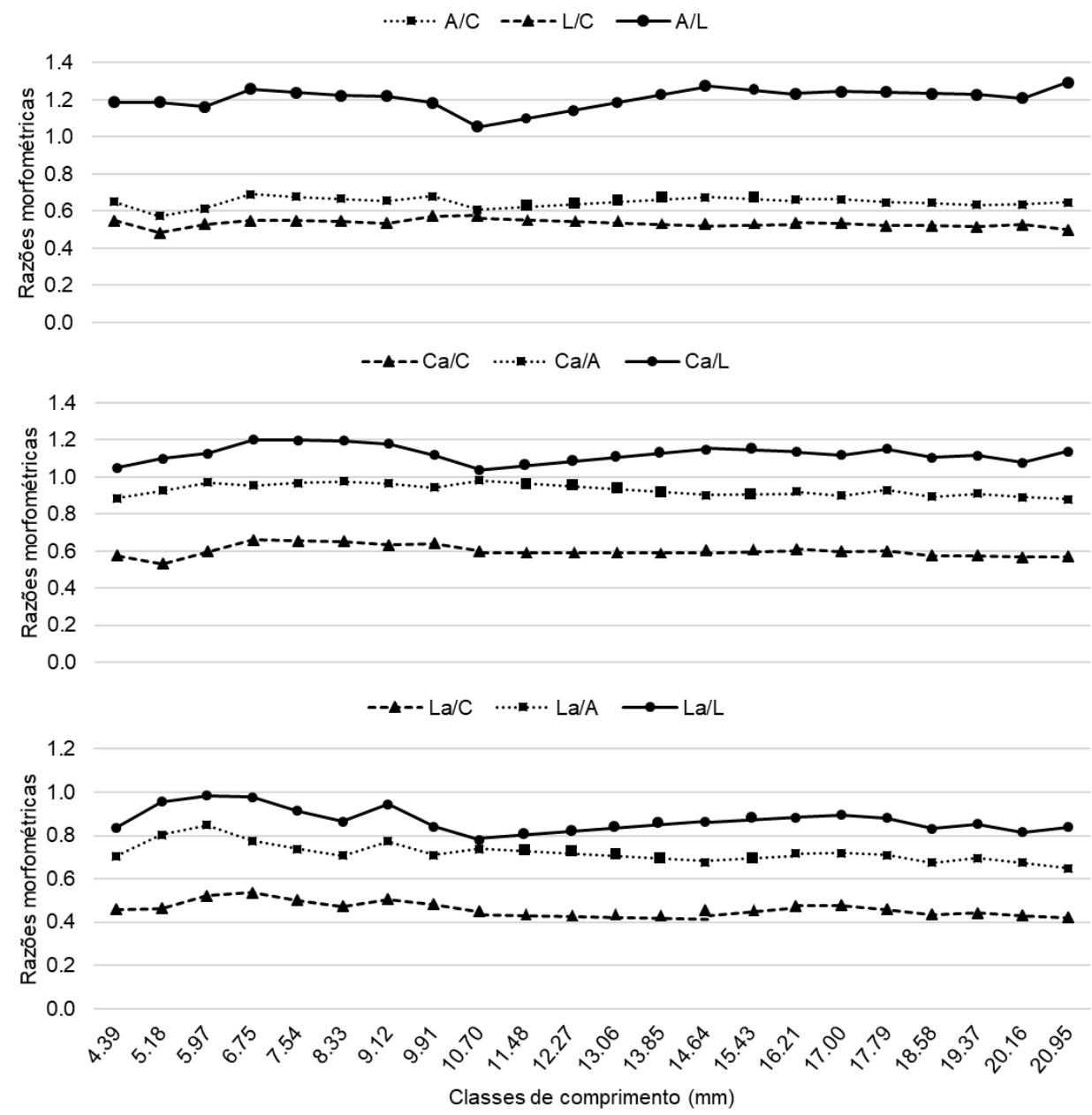

Figura 4. Variação das razões morfométricas ao longo do desenvolvimento de Littoraria flava. Legenda: comprimento (C), largura (L), altura (A), comprimento da abertura (Ca) e largura da abertura (La) da concha. 
As variações na forma da concha de gastrópodes litorinídeos é relatado na literatura científica. Mill \& Grahame (1995) encontraram em seu estudo com Littorina saxatilis (Olivi, 1792), que as variações na forma da concha se devem as variáveis ambientais. Os autores destacam ainda que a principal diferença é evidenciada nas medidas da abertura da concha. Em contrapartida, Moutinho \& Alves-Costa (2000) não encontraram variação na forma da concha em uma comparação entre a medida da abertura e o comprimento da concha de $L$. flava.

Como mencionado, as variáveis ambientais atuam determinando o crescimento dos gastrópodes litorinídeos (Janson 1982). Além disso, atuam nos aspectos populacionais destes moluscos (Catesby \& McKillunp 1998; Cardoso et al. 2007), principalmente pelo ambiente no qual habitam (Fletcher 1995). Neste sentido, o conhecimento da morfologia da concha (Simone 1998), bem como seus aspectos morfométricos (como neste presente estudo), são importantes para uma análise de anomalias na espécie devido a perturbações ambientais.

Por meio deste estudo, determinou-se que as relações morfométricas do gastrópode Littoraria flava são classificadas como "extremamente fortes e positivas" entre as medidas externas e entre as medidas externas e abertura da concha. Adicionalmente, por meio da análise de IEF, verificou-se que $L$. flava apresentou crescimento proporcional ao longo de seu desenvolvimento até a fase adulta.

\section{Agradecimentos}

A Coordenação de Aperfeiçoamento de Pessoal de Nível Superior (CAPES) pela bolsa de pesquisa conferida ao autor R. A. das Chagas. Aos revisores pelas considerações que contribuíram para enriquecer o manuscrito.

\section{Referências}

Andrade S.C.S., Magalhães C.A. \& Solferini V.N. (2003) Patterns of genetic variability in Brazilian Littorinids (Mollusca): a macrogeographic approach. Journal of Zoological Systematics and Evolutionary Research, 41: 249-255. https://doi.org/10.1046/j.1439-0469.2003.00227.x

Barros M.R.F. \& Chagas R.A.d. (2019) Use of mollusks in zoohandicraft manufacturing in the Amazon Region. Brazilian Journal of Biological Sciences, 6(12): 263-269. https://doi.org/10.21472/bjbs.061224

Bauer B., Fioroni P., Ide I., Liebe S., Oehlmann J., Stroben E. \& Watermann B. (1995) TBT effects on the female genital system of Littorina littorea: a possible indicator of tributyltin pollution. Hydrobiologia, 309: 15-27. https://doi.org/10.1007/BF00014468

Cardoso R.S., Costa S.D. \& Loureiro V.F. (2007) Mating behaviour of the marine snail Littoraria flava (Mollusca: Caenogastropoda) on a boulder shore of south-east Brazil. Journal of the Marine Biological Association of the United Kingdom, 87: 947-952. https://doi.org/10.1017/S0025315407053210

Catesby S.M. \& McKillunp S.C. (1998) The importance of crevices to the intertidal snail Littoraria articulata (Philippi) in a tropical mangrove forest. Hydrobiologia, 367: 131-138. https://doi.org/10.1023/A:1003271915241

Chagas R.A.d., Barros M.R.F. \& Bezerra A.M. (2018a) Morfometria da concha do gastrópode invasor Melanoides tuberculata (Müller, 1774) (Gastropoda, Thiaridae). Acta of Fisheries and Aquatic Resources, 6(1): 10-16. http://dx.doi.org/10.2312/Actafish.2018.6.1.10-16

Chagas R.A.d., Barros M.R.F., Miranda A.P.S. \& Santos W.C.R.d. (2018b) Shell morphometry of gastropod Littoraria flava (King, 1832) collected on a rocky shore in Balneario Camboriu, State of Santa Catarina, Brazil. Universidade Federal Rural da Amazônia, Brazil. PANGAEA - Data Publisher for Earth \& Environmental Science. https://doi.pangaea.de/10.1594/PANGAEA.896325

Chapman M.G. (1995) Spatial patterns of shell shape of three species of co-existing littorinids snails in new South Wales, Australia. Journal of Molluscan Studies, 61: 141-162. https://doi.org/10.1093/mollus/61.2.141 
Doyle D., Gammell M.P. \& Nash R. (2018) Morphometric methods for the analysis and classification of gastropods: a comparison using Littorina littorea. Journal of Molluscan Studies, 84(2): 190-197. https://doi.org/10.1093/mollus/eyy010

Ferreira-Jr A.L., Carvalho I.F.P., Absher T.M. \& Cristo S.W. (2014) Reprodução e potenciais impactos sbre Littorina flava (Neogastropoda: Littorinidae) na Ilha do Mel, Paraná. Brazilian Journal of Aquatic Science and Technology, 18(2): 53-57. http://dx.doi.org/10.14210/bjast.v18n2.p53-57

Fletcher C.R. (1995) Microgeographical variation in shell strength in the flat periwinkles Littorina obtusata and Littorina mariae. Hydrobiologia, 309: 73-87. https://doi.org/10.1007/BF00014474

Gaspar M.B., Santos M.N. \& Vasconcelos P. (2001) Weight-length relationships of 25 bivalve species (Mollusca: Bivalvia) from the Algarve coast (southern Portugal). Journal of the Marine Biological Association of the United Kingdom, 81: 805-807. https://doi.org/10.1017/S0025315401004623

Gil G.M., Troncoso J.S. \& Thomé J.W. (2007) Shell shape stabilization indicator (IEF): Handling and optimization of bivalves mollusks exploitation. Porto Alegre: Edição do autor. $152 \mathrm{p}$.

Hammer Ø. (2020) PAST - Palaeontological statistics. Version 4.0. Natural History Museum: University of Oslo. $262 \mathrm{p}$.

Janson K. (1982) Genetic and environmental effect on the growth rate of Littorina saxatilis. Marine Biology, 69(1): 73-78. https://doi.org/10.1007/BF00396963

Meirelles C.A.O. \& Matthews-Cascon H. (2003) Relations between shell size and radula size in marine prosobranchs (Mollusca: Gastropoda). Thalassas, 19(1): 45-53.

Mill P.J. \& Grahame J.W. (1995) Shape variation in the rough periwinkle Littorina saxatilis on the west and south coasts of Britain. Hydrobiologia, 309: 61-71. https://doi.org/10.1007/BF00014473

Moutinho P.R.S. \& Alves-Costa C.P. (2000) Shell size variation and aggregation behavior of Littoraria flava (Gastropoda: LIttorinidae) on a Southeastern Brazilian shore. The Veliger, 43(3): 277-281.

Poutiers J.M. (2016) Gastropods (p. 907-1115). In: Carpenter K.E. \& De Angelis N. (Eds). The living marine resources of the Western Central Atlantic. Volume 2: Bivalves, gastropods, hagfishes, sharks, batoid fishes, and chimaeras. Rome: FAO. 1509 p.

Reid D.G., Dyal P. \& Williams S.T. (2012) A global molecular phylogeny of 147 periwinkle species (Gastropoda, Littorininae). Zoologica Scripta, 41(2): 125-136. https://doi.org/10.1111/j.1463-6409.2011.00505.x

Reid D.G., Lal K., Mackenzie-Dodds J., Kaligis F., Littlewood D.T.J. \& Williams S.T. (2006) Comparative phylogeography and species boundaries in Echinolittorina snails in the central Indo-West Pacific. Journal of Biogeography, 33(6): 990-1006. https://doi.org/10.1111/j.13652699.2006.01469.x

Rios E.C. (2009) Compendium of Brazilian Sea Shells. Rio Grande, RS: Evangraf. 676 p.

Rodrigues W.C. (2010) Estatística Aplicada. $8^{\circ}$ edição. Paracambi: W.C. Rodrigues. 70 p.

Simone L.R.L. (1998) Morphological study on Littorina flava (King \& Broderip) from Brazil (Caenogastropoda, Littorinidae). Revista Brasileira de Zoologia, 15(4): 875-887. http://dx.doi.org/10.1590/S0101-81751998000400005

Vianna B.S., Giodiano F., Dominguez P.S.A. \& Barella M.R. (2014) Análise comportamental do deslocamento do caramujo Littorina flava em costão rochoso da Praia Barra do Una, Peruíbe SP. UNISANTA BioScience, 3(2): 101-107.

Walker T.N. \& Grahame J.W. (2011) Shell shape variation and fitness variables in the gastropod Littorina saxatilis. Marine Ecology, 430: 103-111. https://doi.org/10.3354/meps09122

Zar J.H. (2010) Biostatistical Analysis. $5^{\circ}$ edition. New Jersey: Prentice Hall. 960 p. 